\title{
Argininosuccinate neurotoxicity and prevention by creatine in argininosuccinate lyase deficiency: an in vitro study in rat 3D organotypic brain cell cultures
}

Carmen Diez-Fernandez ${ }^{1}$, Damian Hertig ${ }^{2,3,4}$, Marc Loup ${ }^{5}$, Gaelle Diserens ${ }^{3}$, Hugues Henry ${ }^{5}$, Peter Vermathen ${ }^{3}$, Jean-Marc Nuoffer ${ }^{2}$, Johannes Häberle ${ }^{1 *}$, Olivier Braissant ${ }^{{ }^{*}}$

${ }^{1}$ Division of Metabolism and Children's Research Center, University Children's Hospital Zurich, Zurich, Switzerland.

${ }^{2}$ Division of Pediatric Endocrinology, Diabetology and Metabolism and University Institute of Clinical Chemistry, Inselspital, University Hospital, University of Bern, Bern, Switzerland

${ }^{3}$ AMSM, Departments of Radiology and Biomedical Research, University of Bern, Bern, Switzerland.

${ }^{4}$ Graduate School for Cellular and Biomedical Sciences, University of Bern, Bern, Switzerland

${ }^{5}$ Service of Clinical Chemistry, University Hospital of Lausanne, Lausanne, Switzerland.

* Equal contribution of $\mathrm{JH}$ and $\mathrm{OB}$.

Corresponding authors:

$\underline{\text { Johannes Häberle }}$

Division of Metabolism and Children's Research Center, University Children's Hospital Zurich, 8032 - Zurich, Switzerland Tel: +41 442667342

e-mail: Johannes.haeberle@,kispi.uzh.ch

\section{Olivier Braissant}

Service of Clinical Chemistry, University Hospital of Lausanne

1011 - Lausanne, Switzerland Tel: +41 213144152

e-mail: Olivier.Braissant@.chuv.ch

Word count summary: 249

This article has been accepted for publication and undergone full peer review but has not been through the copyediting, typesetting, pagination and proofreading process which may lead to differences between this version and the Version of Record. Please cite this article as doi: $10.1002 /$ jimd.12090 
Word count text:

3946

Number of figures:

4

Number of tables:

1 (as supplementary material)

This article is protected by copyright. All rights reserved. 


\begin{abstract}
The urea cycle disorder (UCD) argininosuccinate lyase (ASL) deficiency, caused by a defective ASL enzyme, exhibits a wide range of phenotypes, from life-threatening neonatal hyperammonemia to asymptomatic patients, with only the biochemical marker argininosuccinic acid (ASA) elevated in body fluids. Remarkably, even without ever suffering from hyperammonemia, patients often develop severe cognitive impairment and seizures. The goal of this study was to understand the effect on the known toxic metabolite ASA and the assumed toxic metabolite guanidinosuccinic acid (GSA) on developing brain cells, and to evaluate the potential role of creatine $(\mathrm{Cr})$ supplementation, as it was described protective for brain cells exposed to ammonia.
\end{abstract}

We used an in vitro model, in which we exposed 3D organotypic rat brain cell cultures in aggregates to different combinations of the metabolites of interest at two time points (representing two different developmental stages). After harvest and cryopreservation of the cell cultures, the samples were analysed mainly by metabolite analysis, immunohistochemistry and western blotting.

ASA and GSA were found toxic for astrocytes and neurons. This toxicity could be reverted in vitro by Cr. As well, an anti-apoptotic effect of ASA was revealed, which could contribute to the neurotoxicity in ASL deficiency. Further studies in human ASL deficiency will be required to understand the biochemical situation in the brain of affected patients, and to investigate the impact of high or low arginine doses on brain $\mathrm{Cr}$ availability. In addition, clinical trials to evaluate the beneficial effect of $\mathrm{Cr}$ supplementation in ASL deficiency would be valuable. 
This article is protected by copyright. All rights reserved. 


\section{Take-home message}

Argininosuccinic acid and guanidinosuccinic acid, both metabolites present in ASL deficiency, are neurotoxic and affect mainly astrocytes but also neurons; creatine can revert this toxicity. These new findings should be investigated in human ASL deficiency to open up a possible novel therapeutic option in this disease.

Key words: argininosuccinate lyase (ASL) deficiency; urea cycle disorders; neurotoxicity; argininosuccinic acid; guanidinosuccinic acid; guanidino compounds; astrocytes; neurons; 3D organotypic brain cell cultures; creatine

\section{Compliance with Ethics Guidelines}

Carmen Diez-Fernandez, Damian Hertig, Marc Loup, Gaelle Diserens, Hugues Henry, Peter Vermathen, Jean-Marc Nuoffer, Johannes Häberle and Olivier Braissant declare that they have no conflict of interest.

This article does not contain any studies with human subjects.

\section{Authors contribution}

CDF, PV, JMN, JH and OB have planned the conception and design of the study. CDF, ML, DH, GD, HH, PV and JMN have performed the experiments. CDF, PV, JMN, JH and OB have performed data analysis. $\mathrm{CDF}, \mathrm{PV}, \mathrm{JMN}, \mathrm{JH}$ and $\mathrm{OB}$ wrote the manuscript and designed 
the figures, which were revised by all authors. All authors agreed to the final version of the manuscript. 


\section{Introduction}

Argininosuccinate lyase (ASL; MIM *608310) catalyzes as part of the urea cycle the breakdown of argininosuccinic acid (ASA) into fumarate and arginine. In the liver, this reaction is essential for ammonia detoxification and arginine synthesis (O'Brien and Barr 1981). In addition, ASL is expressed in many nitric-oxide (NO) producing tissues like skin fibroblasts (O'Brien and Barr 1981), kidney (Ratner and Petrack 1953), small intestine (Wu et al. 1994), heart, brain (neurons and astroglia) (Braissant et al. 1999; Bizzoco et al. 2007), endothelial cells (Erez et al. 2011), muscle and erythrocytes (Tomlinson and Westall 1964). In these cells, ASL is part of the citrulline-NO cycle.

A deficiency of ASL function (ASLD) leads to argininosuccinic aciduria (MIM \#207900), the second most common urea cycle disorder (UCD) with an incidence of $\sim 1: 218,750$ (Summar et al. 2013). It is an autosomal recessive disorder caused by mutations in the $A S L$ gene, which maps to 7q11.21 (O'Brien et al. 1986), spans 17,5 kb, has an open reading frame of $1,392 \mathrm{bp}$ and harbors 16 coding exons. Most mutations reported so far $(>160)$ are missense mutations distributed through exons 2 to 16 (of which exons 3, 4, 6, 7 and 9 harbor $41 \%$ of the mutations, while only accounting for $25 \%$ of the total $A S L$ sequence) (Balmer et al. 2014). ASL functions as a homotetramer constituted by four $51 \mathrm{kDa}$ monomers (Simard et al. 1986). Each monomer consists of three domains: domains 1 and 3 have a similar helix-turn-helix structure while domain 2 is formed by nine helices, three of which are involved in dimerization with another ASL monomer (Turner et al. 1997). Furthermore, there are three 
highly conserved separated regions, which upon tetramerization come together and form an active site where each region contributes by a different monomer (Sampaleanu et al. 2001). ASLD can develop as either a severe neonatal onset form with life-threatening hyperammonemia or as a late onset with episodic hyperammonemia and long-term complications including liver dysfunction, neurocognitive deficits, arterial hypertension, behavioural abnormalities and learning disabilities. These long-term complications can occur even in absence of observed hyperammonemia crises. In fact, patients with ASLD have, in comparison to other UCDs, a remarkable poor outcome. This indicates that tissue-specific absence of ASL may cause these symptoms. In addition to the aforementioned clinical presentations, there are asymptomatic ASLD patients, possibly detected through newborn screening, that only show biochemical manifestations.

The hallmark of the disease is the accumulation of ASA in plasma and urine (Baruteau et al. 2017), a metabolite normally not detected (Häberle and Rubio 2014). The biochemical alterations in ASLD patients include increased plasma citrulline and decreased arginine (Häberle and Rubio 2014; Roze et al. 2007), which may affect all related metabolites: NO, polyamines, proline, glutamate, creatine $(\mathrm{Cr})$ and agmatin. In fact, the loss of ASL led to a global NO deficiency in mice (Erez et al. 2011). Furthermore, it has been suggested that the decreased arginine levels cause a decoupling of NO synthase (NOS), which leads to accumulation of free radicals. These free radicals have the potential to attack accumulating ASA, hereby generating guanidinosuccinic acid (GSA) (Aoyagi et al. 1999), which could contribute to the neurotoxicity observed in ASLD patients. Although GSA has not yet been measured in brain, another guanidino compound, guanidinoacetate (GAA; $\mathrm{Cr}$ synthesis 
pathway intermediate) was found elevated in the brain of ASLD patients (Baruteau et al. 2017; van Spronsen et al. 2006), while in plasma and urine GAA was within normal ranges (Häberle and Rubio 2014). As well, a secondary Cr deficiency was suggested in these patients, at least in those not yet treated with arginine (Arias et al. 2004). It would be relevant to discern if such Cr deficiency is observed globally (plasma, urine, brain), or restricted to CNS as was the case for GAA (Baruteau et al. 2017; van Spronsen et al. 2006).

In contrast to other UCDs and sometimes without ever suffering from hyperammonemia, ASLD patients often develop a severe neurologic disorder including mental retardation and seizures (Baruteau et al. 2017; Nagamani et al. 2012). Consequently, our aims were to understand the effects of those metabolites that likely accumulate in ASLD, ASA and GSA, on the developing brain cells. In addition, we wanted to test if $\mathrm{Cr}$, proposed in trials to treat neurodegenerative disorders and found deficient in ASLD patients, had protective effects. To do so, we exploited an in vitro model, already used to study the effect of accumulating toxic metabolites in other metabolic diseases (Hanna-El-Daher et al. 2015; Jafari et al. 2013a), in which we exposed 3D organotypic rat brain cell cultures in aggregates to different combinations of the metabolites of interest at two time points (representing two different developmental stages). This model reproduced the production and accumulation of these metabolites during a metabolic crisis. After harvest and cryopreservation of the cell cultures, a thorough analysis of the samples was performed, including metabolite analysis, immunohistochemistry and western blotting. 


\section{Materials and Methods}

\section{D organotypic cultures of developing brain cells}

Handling of pregnant rats (Sprague-Dawley 300 g, Charles River) was done following all institutional and national guidelines for the care and use of laboratory animals (Swiss Academy for Medical Science rules). At E15.5 days the embryos were dissected to prepare 3D primary cultures of brain cells in aggregates. For this purpose, the whole brains were mechanically dissociated and cultured under continuous gyratory agitation $(80 \mathrm{rpm})$ in a serum-free and chemically defined medium, as previously described (Braissant et al. 2002). Spontaneously, all brain cells (including neurons, astrocytes and oligodendrocytes), reaggregate in an organized $3 \mathrm{D}$ network, resembling the tissue-specific pattern of the in vivo brain. These 3D cultures are thus considered as organotypic brain cell cultures, which can generate their own arginine and NO, thanks to the expression of NOS, argininosuccinate synthetase (ASS) and ASL (Braissant et al. 1999).

To study the effect of metabolites accumulating in ASLD and to test the protective effect of specific molecules, cultures were treated by repeated administration of different combinations of compounds (see below), at two different time points representing different developmental stages: from day in vitro (DIV) 5 to DIV13, representing a neonatal brain, and from DIV14 to DIV22, representing an early infant brain (Figure 1A). Brain cell cultures were exposed to the following metabolites: argininosuccinate (ASA) at 1, 10, 100 or $1000 \mu \mathrm{M}$; GSA at 1, 10, 30 or $100 \mu \mathrm{M} ; \mathrm{NH}_{4} \mathrm{Cl}$ at $5 \mathrm{mM} ; \mathrm{Cr}$ at $1 \mathrm{mM}$. Aggregates were harvested at DIV13 and 
DIV22 by sedimentation, followed by three rinsing with cold PBS, and either frozen in liquid nitrogen for metabolite (liquid chromatography coupled to tandem-mass spectrometry, LC/MS-MS) and protein (western blotting) analysis, or embedded in cryo-medium (TissueTek O.C.T., Digitana) and frozen in liquid nitrogen-cooled isopentane for immunohistological analysis. Samples were kept at $-80^{\circ} \mathrm{C}$ until use. Culture medium was harvested with every medium change (every 3 days until DIV14; every 2 days from DIV14 to 22; Figure 1A) and kept at $-80{ }^{\circ} \mathrm{C}$ for metabolite analysis by LC/MS-MS.

\section{Antibodies, western blotting and immunofluorescence}

For western blotting, primary antibodies against the following proteins were used: Actin (1:3000, A1978, Sigma), ASS (1:1000, ARP41366_T100, Aviva Systems), ASL (1:200, E-5 sc-374353, Santa Cruz Biotechnology), cleaved caspase-3 (1:200, 9664) and caspase-3 (1:1000, 9665) (Cell Signaling Technology), glial fibrillary acidic protein (GFAP, 1:1000, clone GA5, MAB 360, Millipore), medium weight neurofilament (NFM, 1:1000, clone NF09, sc51683, Santa Cruz technology), phosphorylated NFM (p-NFM, 1:500, clone NN18, MAB 5254, Millipore) and NOS1 (1:500, A-11 sc-5302, Santa Cruz Biotechnology). Secondary antibodies were horse radish peroxidase-conjugated goat anti-rabbit (1:3000, sc2301 Santa Cruz Biotechnology) and anti-mouse (1:3000, sc-516102 Santa Cruz Biotechnology). For immunofluorescence, primary antibodies against the following proteins were used: GFAP (1:100), myelin basic protein (MBP, 1:100) and cleaved caspase-3 (1:100). Secondary antibodies were goat anti-mouse, anti-rabbit or donkey anti-goat IgG labeled with Alexa Fluor® 555 (red) (Life Technologies). Western blotting was performed as described 
(Hanna-El-Daher et al. 2015), loading $15 \mu \mathrm{g}$ total protein in each gel lane. After $1^{\text {st }}$ development, blots were stripped, re-probed and developed for the respective other genes. Images were taken with a Molecular Imager ChemiDoc XRS+ (BioRad) and quantified with the Image $\mathrm{Lab}^{\mathrm{TM}}$ Software (BioRad). For immunofluorescence, $16 \mu \mathrm{m}$ cryosections of aggregates were prepared and probed with the respective antibodies as described, including using Tyramide Signal Amplification (TSA, Molecular Probes) system for cleaved and total caspase 3 (Hanna-El-Daher et al, 2015). Sections were observed and digitized on an Olympus BX50 microscope equipped for red fluorescence; images were processed using the Cell Sens Imaging Software (Olympus).

\section{Measurement of metabolites and lactate dehydrogenase in the culture media}

Glucose, lactate and lactate dehydrogenase (LDH) were measured in culture media harvested at DIV13 and DIV22 using a COBAS 8000 analyzer (Roche, Switzerland). Ammonium in culture media was measured using an INTEGRA 200 analyzer (Roche, Switzerland). Intracellular concentrations of amino acids, as well as $\mathrm{Cr}$ and GAA, were measured in lysates of brain cell aggregates by LC/MS-MS as previously described (Hanna-El-Daher et al. 2015).

Nuclear magnetic resonance (NMR) spectroscopy measurements of intracellular metabolites were performed as previously described (Diserens et al. 2018).

\section{Statistical analysis}


Data were expressed as mean \pm standard error of the mean (SEM). Statistical significance of the differences between various conditions was determined by Student's $t$-test (one way analysis of variance); $\mathrm{p}<0.05$ was considered significant: ${ }^{+} \mathrm{p}<0.05 ;{ }^{++} \mathrm{p}<0.01 ;{ }^{++} \mathrm{p}<0.001$. 


\section{Results}

\section{Biochemical characterization of the ASA-exposed brain cell cultures}

\section{Glucose and lactate in culture medium as indicators of metabolic activity}

In controls, glucose levels in immature cultures (DIV13) were higher than that of the later stage (DIV22) (Figure 1B). Upon addition of metabolites (ASA, GSA, $\mathrm{NH}_{4} \mathrm{Cl}, \mathrm{Cr}$ ), glucose did not change significantly in any condition in the immature culture. In contrast, at later stage, addition of $100 \mu \mathrm{M}$ ASA caused a significant decrease in glucose levels; when ASA and $\mathrm{NH}_{4}{ }^{+}$were combined, glucose levels were even lower; and in presence of $100 \mu \mathrm{M}$ GSA, glucose became undetectable (Figure 1B). In parallel, a significant increase in lactate was observed in controls at DIV22 with respect to that at DIV13 (Figure 1B). When immature cultures were stressed with $\mathrm{NH}_{4}{ }^{+}$, lactate increased as well, as already described (Braissant et al. 2002). In contrast, lactate levels in the more mature stage remained essentially unchanged in every condition, except under $100 \mu \mathrm{M}$ GSA which presented a $60 \%$ decrease in lactate, suggesting severe suffering of the cultures under this condition (see below). NMR spectroscopy measurements confirmed the increased glucose consumption at DIV22 with elevated intracellular glycolytic metabolites (glucose-1-phosphate and lactate) and the effect of $100 \mu \mathrm{M}$ GSA on glucose and lactate. Addition of combined ASA, GSA with or without $\mathrm{Cr}$ did not lead to a significantly different metabolomic pattern defined by 39 intracellular metabolites detected by NMR (Supplementary Table 1). 


\section{Stage-dependent metabolization of ammonium}

While developing cultures (DIV13) showed $\mathrm{NH}_{4}{ }^{+}$levels of $100 \mu \mathrm{M}$ (Figure 1C) in controls (as well as ASA-only and GSA-exposed conditions), mature cultures (DIV 22) showed mean $\mathrm{NH}_{4}{ }^{+}$levels of $40 \mu \mathrm{M}$ (Figure 1C), illustrating the maturation of astrocytes expressing glutamine synthetase (the enzyme converting glutamate and $\mathrm{NH}_{4}{ }^{+}$to glutamine). These data are in accordance with our previous studies (Braissant et al. 2002). Accordingly, young cultures exposed to $5 \mathrm{mM} \mathrm{NH}_{4} \mathrm{Cl}$ showed extremely high levels of $\mathrm{NH}_{4}{ }^{+}$in culture medium (>2 mM), while mature cultures exposed to $\mathrm{NH}_{4}{ }^{+}$were able to metabolize it and showed levels of $\mathrm{NH}_{4}{ }^{+}$between 100 and $200 \mu \mathrm{M}$ (Figure 1C).

\section{Lactate dehydrogenase (LDH) release as indicator of cell death}

While in all but one conditions tested LDH remained barely detectable in culture media, 100 $\mu \mathrm{M}$ GSA exposure in mature cultures led to an important release of $\mathrm{LDH}$, indicating suffering and massive cell death under this condition (Figure 1D). This was also reflected by the undetectable level of glucose at DIV22 of these GSA-exposed mature cultures, and by the severe reduction of gene expression for specific brain cell markers (e.g. GFAP for astrocytes; NFM/p-NFM for neurons; Figure 2). Interestingly, $100 \mu \mathrm{M}$ GSA exposure in immature cultures did not provoke this severe condition.

\section{Differential toxicity of ASA and GSA exposure on brain cell types}


While low levels of ASA exposure (e.g. 1 and $10 \mu \mathrm{M}$ ) did not appear to affect both developing (exposure DIV 5 to 13) and mature (exposure DIV 14 to 22) brain cells (see Figure 2), higher doses of ASA (e.g. $100 \mu \mathrm{M}$ and $1 \mathrm{mM}$ ) showed differential neurotoxicity depending on the brain cell types. Similarly, a dose-dependent assay was first performed to evaluate the toxicity of GSA. While 1 and $10 \mu \mathrm{M}$ GSA did not affect brain cells much (see Figure 2), $100 \mu \mathrm{M}$ proved to be toxic and led to the rapid culture death (illustrated in Figure 1D by massive LDH release in culture medium). We thus chose an intermediate dose of 30 $\mu \mathrm{M}$ GSA in co-exposure with $100 \mu \mathrm{M}$ ASA to evaluate the combined neurotoxicity of both ASA and GSA.

Neurons were affected by ASA exposure, in particular in developing cultures (DIV 13) exposed to $100 \mu \mathrm{M}$ ASA, as shown by decrease of both NFM and p-NFM (Figure 2A). 1 $\mathrm{mM}$ Cr co-exposure prevented the ASA-induced NFM and p-NFM decrease. NFM and pNFM expression were not affected by ASA exposure in mature cultures (DIV22) (Figure 2A). While co-exposure to $30 \mu \mathrm{M}$ GSA (with ASA $100 \mu \mathrm{M}$; plus or minus $1 \mathrm{mM} \mathrm{Cr}$ ) did not affect NFM and pNFM in mature cultures, $30 \mu \mathrm{M}$ GSA interestingly appeared as protective as $1 \mathrm{mM} \mathrm{Cr}$ in developing cultures. As for astrocytes, $100 \mu \mathrm{M}$ GSA strongly decreased pNFM expression in both stages of cultures. Co-exposure to $5 \mathrm{mM} \mathrm{NH}_{4} \mathrm{Cl}$ with $100 \mu \mathrm{M}$ ASA strongly affected NFM and p-NFM expression in developing and mature cultures, this toxic effect being prevented by $1 \mathrm{mM} \mathrm{Cr}$ co-exposure in mature culture only (Figure $\mathbf{2 A}$ ) Astrocytes appeared the most affected cell type, presenting decreased GFAP expression in both stages, as shown by immunohistochemistry and western blotting (Figure 2B). Coexposure to ammonium $(5 \mathrm{mM})$ aggravated toxicity in both stages, showed by further 
decrease in GFAP expression. Cr supplementation $(1 \mathrm{mM})$ prevented ASA toxicity on GFAP (for both $100 \mu \mathrm{M}$ and $1 \mathrm{mM}$ ASA) with or without co-exposure to ammonium (Figure 2B). While 1 and $10 \mu \mathrm{M}$ of GSA exposure did not appear to affect astrocytes, $100 \mu \mathrm{M}$ GSA led to almost complete repression of GFAP expression in mature cultures. $30 \mu \mathrm{M}$ GSA co-exposure did not appear to increase further the $100 \mu \mathrm{M}$ ASA-induced astrocytic toxicity on GFAP. However, the presence of GSA impeached the protective effect of $\mathrm{Cr}$ supplementation, nevertheless demonstrating some GSA toxicity on astrocytes, both in developing and mature cultures (Figure 2B).

In contrast, oligodendrocytes were not sensitive to ASA, at both $100 \mu \mathrm{M}$ and $1 \mathrm{mM}$ and in both stages, while ammonium co-exposure was detrimental to MBP expression in immature culture. Cr supplementation partially reverted ammonium toxicity on MBP expression in these immature cultures. No effect of $30 \mu \mathrm{M}$ GSA and $100 \mu \mathrm{M}$ ASA co-exposure was observed on oligodendrocytes (Figure 2C).

\section{Anti-apoptotic effect of ASA exposure, and ammonium-induced apoptosis}

$100 \mu \mathrm{M}$ ASA exposure appeared anti-apoptotic in developing cultures, as shown by both immunohistochemistry and western blotting for cleaved caspase 3 , while total caspase 3 remained stable. This effect was not observed in mature cultures. $30 \mu \mathrm{M}$ GSA co-exposure with $100 \mu \mathrm{M}$ ASA did not alter the ASA-induced anti-apoptotic effect, while $1 \mathrm{mM} \mathrm{Cr}$ coexposure, with or without GSA, further amplified the ASA-induced anti-apoptotic effect

(Figure 3). Ammonium co-exposure with $100 \mu \mathrm{M}$ ASA strongly stimulated apoptosis in 
brain cells, in particular at the younger stage (DIV13). Interestingly, this effect was prevented by $\mathrm{Cr}(1 \mathrm{mM})$ co-exposure (Figure 3).

\section{Effects of ASA, GSA, ammonium and $\mathrm{Cr}$ on the citrulline-NO and $\mathrm{Cr}$ pathways}

With the aim of better understanding the mechanisms of ASA-induced toxicity to brain cells, and prevention by $\mathrm{Cr}$ supplementation, we decided to evaluate whether the expression of enzymes processing ASA (e.g. ASS and ASL; as well as NOS1, making together the citrulline-NO pathway) were affected by ASA exposure, with or without ammonium coexposure or $\mathrm{Cr}$ supplementation. These three proteins showed a strong decreased expression in younger cultures (DIV 13) at $100 \mu \mathrm{M}$ ASA exposure (Figure 4A), while expression of ASS, ASL and NOS1 was barely affected in the mature cultures (Figure 4B). This effect on developing cells was prevented by $1 \mathrm{mM} \mathrm{Cr}$ supplementation. Intriguingly, $30 \mu \mathrm{M}$ GSA coexposure with $100 \mu \mathrm{M}$ ASA had the same protective effect as $1 \mathrm{mM} \mathrm{Cr}$. Ammonium coexposure (with $100 \mu \mathrm{M}$ ASA) further decreased ASS, ASL and NOS1 expression in younger cultures; Cr supplementation being protective only for NOS1 (Figure 4A). In mature cultures (DIV22), a detrimental effect of ammonium exposure was observed on NOS1 only (without any effect of ASA co-exposure), which was prevented also by $\mathrm{Cr}$ supplementation (Figure 4B).

We then evaluated whether the above-described effects on the citrulline-NO pathway could also affect the Cr synthesis pathway, as arginine, produced by the ASL reaction and entering AGAT reaction, is the limiting substrate for Cr synthesis. We thus measured, in the younger cultures (DIV13), the intracellular levels of arginine, GAA (the intermediate between AGAT 
and GAMT reactions) and Cr by LC/MS-MS (Figure 4C). We show here that while $1 \mu \mathrm{M}$ ASA appeared stimulatory on arginine, GAA and Cr levels, $100 \mu \mathrm{M}$ ASA induced a $20 \%$ decrease of the three metabolites. Ammonium co-exposure induced a 25\% decrease of both Arg and $\mathrm{Cr}$ at 1 and $10 \mu \mathrm{M}$ ASA, while no further decrease was observed at $100 \mu \mathrm{M}$ ASA. Ammonium co-exposure strongly decreased GAA levels. $\mathrm{Cr}$ supplementation strongly increased intracellular levels of $\mathrm{Cr}(+80 \%)$ but decreased GAA, and no change in $\mathrm{Arg}$ concentration could be observed. GSA did not affect intracellular levels of Arg, $\mathrm{Cr}$ and GAA, even at the highest concentration tested $(100 \mu \mathrm{M})$. 


\section{Discussion}

Patients with ASLD often show worse neurological outcome than other UCDs (Erez 2013; Rüegger et al. 2014) and the reasons for this are not clear. We aimed here at a better understanding of this phenomenon by investigating the effect of the known toxic metabolite ASA and the assumed toxic metabolite GSA on brain cells, and by evaluating the role of $\mathrm{Cr}$, as it was described protective for brain cells exposed to ammonia (Braissant 2010).

We first investigated the overall biochemical situation of the 3D cell cultures in the presence of toxic metabolites. Apart of the normal increase of metabolic activity between immature and mature cultures (increase of glucose consumption and lactate release) and the already described metabolic stress induced by ammonium exposure (Braissant et al. 2002), no evident metabolic stress was observed through extracellular glucose, lactate, ammonium or LDH measures under ASA or GSA exposure, with the exception of high $100 \mu \mathrm{M}$ GSA exposure which appeared extremely toxic to mature cultures (lactate strongly decreased; LDH release; protein expression extinction for GFAP and NFM for example). While GSA levels in human patients are not known, an increase of GSA seems likely given the known cerebral accumulation of another guanidino compound, guanidinoacetate, in ASLD (van Spronsen et al. 2006). GSA toxicity was further underlined by a decreased expression of astrocytic and neuronal markers in immunohistochemistry. The toxic effect of GSA was however not seen in all conditions. For instance, immature brain cells were more tolerant towards this metabolite and the reason for this remains unknown. We did not study in detail the 
consequences of high $\mathrm{NH}_{4}{ }^{+}$since this was previously thoroughly characterized in the same system (Braissant 2010; Braissant et al. 2008; Braissant et al. 2002).

We next wanted to know what cell types were mostly affected by ASA and GSA. This was evaluated in experiments with ASA alone or in co-exposure with GSA. Hereby, we found astrocytes being the most affected brain cell type, as shown by decreased GFAP expression in early and mature stages. This pattern was caused by ASA alone, and could be aggravated by addition of $\mathrm{NH}_{4}^{+}$. Interestingly, $\mathrm{Cr}$ could prevent this ASA toxicity, even when cells were exposed to high ASA doses $(1 \mathrm{mM})$. The same was seen upon exposure to $30 \mu \mathrm{M}$ GSA, while lower amounts GSA did not further increase ASA toxicity.

In contrast, the effect of ASA alone or together with GSA was less severe but still clear in neurons at early stages. Again, Cr could prevent this ASA induced toxicity, and similarly when ASA was given together with $\mathrm{NH}_{4}{ }^{+}$. The least affected cell type were oligodendrocytes, which only showed decreased MBP expression if ASA plus $\mathrm{NH}_{4}^{+}$were given, suggesting an ammonium-only effect, supporting previous results (Cagnon and Braissant 2009). The pattern of affected brain cells possibly reflects levels of expression: ASL is expressed by neurons and astrocytes, while, in contrast, oligodendrocytes do not express ASL. Thus, due to the presence of ASL within neurons and astrocytes, ASA exposure might primarily affect these two cell types, for example through inhibition feedback loops on ASL expression, as well as on disturbance of the citrulline-NO cycle/NO production.

Taken together, we prove here that ASA and GSA are both toxic to astrocytes, and to a lesser extent to neurons, with some dose dependent effect. While ASA is a proven metabolite in 
ASLD, there is not yet enough evidence for increased GSA levels in brains of ASLD patients, although this can be assumed based on basic biochemistry. However, investigations in patients should be performed to measure GSA and hereby substantiate the role of GSA as an important factor in the pathophysiology of ASLD.

The cause of the ASA and GSA toxicity remains partly unclear. We aimed at studying a possible impact of toxic metabolites on apoptosis. Surprisingly however, developing brain cells seemed to be protected by an anti-apoptotic effect of ASA (if given at $100 \mu \mathrm{M}$ ). Coexposure with GSA had the same effect, as the addition of $\mathrm{Cr}$. Only when $\mathrm{NH}_{4}{ }^{+}$was added, this effect was reverted and, in contrast, brain cells went into apoptosis, confirming previous studies (Cagnon and Braissant 2008). It is still speculative but this may point towards an antiapoptotic effect of ASA, in which natural apoptosis occurring at these stages is hampered leading to surplus cells and neurological symptoms. The ASA neurotoxicity would then occur through inhibition of naturally and developmentally programmed cell death. An alternative (or additional) cause of ASA and GSA toxicity could be the decreased expression of the citrulline-NO pathway in developing cells. Again, $\mathrm{Cr}$ prevented this effect, as did a coexposure of $30 \mu \mathrm{M}$ GSA and $100 \mu \mathrm{M}$ ASA, while addition of $\mathrm{NH}_{4}{ }^{+}$enhanced the suppressive effect.

Another explanation of the observed ASA toxicity and its prevention by $\mathrm{Cr}$ supplementation may be a change in the $\mathrm{Cr}$ synthesis pathway. ASA alone or in combination with $\mathrm{NH}_{4}^{+}$ caused relevant reduction of the entire $\mathrm{Cr}$ pathway. As described previously, $\mathrm{NH}_{4}^{+}$coexposure strongly decreased GAA levels, most probably through AGAT inhibition (Braissant et al. 2008). While as expected Cr supplementation strongly increased intracellular levels of 
$\mathrm{Cr}(+80 \%)$, no change in intracellular Arg concentration could be observed. Also, as expected, the increase of intracellular $\mathrm{Cr}$ by $\mathrm{Cr}$ supplementation led to a further decrease in GAA through negative feedback regulation on AGAT, as already shown in brain cells (Braissant et al. 2010). In contrast, the Cr pathway was not affected by GSA, even when given at a high concentration $(100 \mu \mathrm{M})$.

In summary, we identify ASA and GSA as toxic metabolites for mainly astrocytes and neurons. This toxicity can be reverted in vitro by $\mathrm{Cr}$. Further, we reveal an anti-apoptotic effect of ASA, which may contribute to the neurotoxicity in ASLD. Our findings are relevant as they may have therapeutic implications. We propose studies in human ASLD to substantiate the biochemical situation (with a focus on ASA and guanidino compounds) in the CNS of affected patients, and to investigate the impact of high or low arginine doses on brain $\mathrm{Cr}$ availability. Lastly, we propose clinical studies aiming at investigating whether $\mathrm{Cr}$ supplementation can be beneficial in ASLD.

\section{Acknowledgements}

This work was financed by grants from the Swiss National Science Foundation (to JH, grants 310030_153196 and 320030_176088; to OB, grant 31003A_175778) and Spendenstiftung Bank Vontobel (to CDF, project "Pathophysiology of ASL Deficiency"). 


\section{Figure legends}

Figure 1: Experimental setting and effects of ASA, GSA, ammonium and Cr exposure on extracellular basic metabolites and LDH. (A) Experimental setting showing the use of immature (DIV 5-13) and mature (DIV 14-22) 3D brain cell cultures in aggregates. Exposure to $\operatorname{ASA}(1,10,100,1000 \mu \mathrm{M})$, GSA $(1,10,30,100 \mu \mathrm{M}), \mathrm{NH}_{4} \mathrm{Cl}(5 \mathrm{mM})$ and $\mathrm{Cr}(1 \mathrm{mM})$ were repeated at each medium change (DIV 5, 8, 11 for immature cultures; DIV 14, 16, 18, 20 for mature cultures); Harvests were done at DIV 13 and 22 respectively. (B-D) Effects of ASA, GSA, $\mathrm{NH}_{4} \mathrm{Cl}$ and $\mathrm{Cr}$ exposure on extracellular glucose and lactate (B), ammonium (C) and LDH (D). Mean \pm SEM; n: 4-5 depending on replicates; significance level as compared to controls: ${ }^{+} \mathrm{p}<0.05 /{ }^{++} \mathrm{p}<0.01 /^{+++} \mathrm{p}<0.001$ (Student's T test).

Figure 2: ASA exposure affects neurons and astrocytes, but not oligodendrocytes.

(A) ASA-induced dysregulation of the neuronal (and axonal) markers NFM/p-NFM, and prevention by $\boldsymbol{C r}$. Western blot analysis of NFM and p-NFM in immature brain cell cultures (left side) shows that $100 \mu \mathrm{M}$ ASA exposure induces a strong inhibition of both p-NFM (normally enriched in axons) and NFM. This effect is prevented by $1 \mathrm{mM} \mathrm{Cr}$ supplementation as well as, interestingly, by $30 \mu \mathrm{M}$ GSA co-exposure, while $100 \mu \mathrm{M}$ GSA exposure alone almost completely abolishes p-NFM expression. $5 \mathrm{mM} \mathrm{NH} \mathrm{NH}_{4}$ co-exposure reinforces ASA-induced neurotoxicity on p-NFM and NFM, and prevents the protective effect of $\mathrm{Cr}$, in contrast to $\mathrm{NH}_{4} \mathrm{Cl}$ exposure alone (Braissant et al. 2002). In contrast, in 
mature cultures (right side), ASA did not show any toxicity for NFM and p-NFM, while GSA at both 10 and $100 \mu \mathrm{M}$ induced a severe decrease of their expression. $5 \mathrm{mM} \mathrm{NH}_{4} \mathrm{Cl}$ coexposure with $100 \mu \mathrm{M}$ ASA strongly inhibited both p-NFM and NFM expression, an effect prevented by $\mathrm{Cr}$ supplementation, in contrast to immature cultures.

(B) ASA-induced dysregulation of the astrocytic marker GFAP, and prevention by Cr. (B)

As shown by immunofluorescence, ASA exposure $(100 \mu \mathrm{M}$ and $1 \mathrm{mM})$ induces a decrease in GFAP expression in both immature and mature brain cell cultures, while $1 \mathrm{mM} \mathrm{Cr}$ coexposure appears to protect astrocytes against ASA toxicity. $5 \mathrm{mM} \mathrm{NH}_{4} \mathrm{Cl}$ co-exposure with $100 \mu \mathrm{M}$ ASA reinforces the ASA-induced toxicity, while Cr supplementation still appears to have a protective effect on GFAP. $30 \mu \mathrm{M}$ GSA co-exposure with $100 \mu \mathrm{M}$ ASA also reinforces the ASA-induced toxicity, while in that case Cr supplementation do not prevent the toxic effects on GFAP expression. By western blot analysis, we show the more pronounced toxicity of $100 \mu \mathrm{M}$ ASA exposure on GFAP expression in immature cultures, as well as the cumulative toxicity of $5 \mathrm{mM} \mathrm{NH}_{4} \mathrm{Cl}$ and prevention by $1 \mathrm{mM} \mathrm{Cr}$ supplementation. Figure $2 \mathrm{~B}$ also shows that $100 \mu \mathrm{M}$ GSA exposure was deleterious to brain cell cultures, particularly for astrocytes on mature cultures in which GFAP expression was almost completely lost.

(C) ASA and GSA exposures do not alter the expression of the oligodendrocytic marker MBP. We show by immunofluorescence that ASA exposure $(100 \mu \mathrm{M}$ and $1 \mathrm{mM})$, plus or minus $30 \mu \mathrm{M}$ GSA or $1 \mathrm{mM}$ Cr supplementation, does not alter the expression of MBP in oligodendrocytes. In contrast, $5 \mathrm{mM}$ co-exposure with $100 \mu \mathrm{M}$ ASA induced a decrease in MBP expression, which was more pronounced in immature cultures (DIV 13). This toxicity 
of $\mathrm{NH}_{4}^{+}$, already observed in oligodendrocytes in this 3D culture system (Cagnon and Braissant 2009), could be partially prevented by Cr supplementation.

For western blotting: Mean \pm SEM; n: 4-5 depending on replicates; significance level as compared to controls: ${ }^{+} \mathrm{p}<0.05 /{ }^{+++} \mathrm{p}<0.001$ (Student's T test). For immunofluorescence: Bar $=$ $100 \mu \mathrm{m}$.

Figure 3: Anti-apoptotic effect of ASA and Cr exposure on brain cells. (A) Cleaved caspase 3 expression by immunofluorescence. ASA exposure $(100 \mu \mathrm{M}$ and $1 \mathrm{mM})$ decreases the level of cleaved caspase 3 in both immature and mature cultures, suggesting a decrease of natural apoptosis occurring in developing brain cells. $30 \mu \mathrm{M}$ GSA co-exposure with $100 \mu \mathrm{M}$ ASA appears to block this anti-apoptotic effect of ASA. $5 \mathrm{mM} \mathrm{NH}_{4} \mathrm{Cl}$ co-exposure with 100 $\mu \mathrm{M}$ ASA strongly stimulates apoptosis, as already observed in the same system for ammonium exposure alone (Cagnon and Braissant 2008). This pro-apoptotic effect of $5 \mathrm{mM}$ $\mathrm{NH}_{4} \mathrm{Cl}$ co-exposure is prevented by $\mathrm{Cr}$ supplementation. Bar: $100 \mu \mathrm{m}$. (B) Western blot analysis of total and cleaved caspase 3. While $100 \mu \mathrm{M}$ ASA exposure shows a nonsignificant tendency to decrease apoptosis (decrease in cleaved caspase 3 expression) in immature cultures, this effect is strongly re-inforced by $\mathrm{Cr}$ supplementation, including under $30 \mu \mathrm{M}$ GSA co-exposure. This last effect was also observed in mature cultures. Mean $\pm \mathrm{SEM}$; $\mathrm{n}$ : 4-5 depending on replicates; significance level as compared to controls: ${ }^{+++} \mathrm{p}<0.001$ (Student's T test). 
Figure 4: Effects of ASA on citrulline-NO and Cr pathways. (A,B) Western blot analysis of ASS, ASL and NOS1 expression in immature, respectively mature brain cell cultures. While no effect of ASA and Cr exposure was observed on ASS, ASL and NOS1 expression in mature cultures (B; DIV 22), $100 \mu \mathrm{M}$ ASA exposure induced a decrease of ASS, ASL and NOS1 expression (A, DIV 13). This effect was prevented by $1 \mathrm{mM} \mathrm{Cr}$ supplementation as well as, interestingly, by $30 \mu \mathrm{M}$ GSA co-exposure (as for p-NFM and NFM, see Figure 2). 5 $\mathrm{mM} \mathrm{NH} \mathrm{Cl}_{4} \mathrm{Cl}$ co-exposure with ASA prevented the protective effect of $\mathrm{Cr}$, except partially for NOS1. This protective effect of $\mathrm{Cr}$ on $\mathrm{ASA}+\mathrm{NH}_{4} \mathrm{Cl}$ toxicity for $\mathrm{NOS} 1$ expression was also observed in mature cultures (B). Mean \pm SEM; n: 4-5 depending on replicates; significance level as compared to controls: ${ }^{+} \mathrm{p}<0.05 /{ }^{+++} \mathrm{p}<0.001$; significance level as compared to ASA $+\mathrm{NH}_{4} \mathrm{Cl}$ exposure: ${ }^{*} \mathrm{p}<0.05$ (Student's T test). (C) LC/MS-MS measure of intracellular arginine, $\mathrm{Cr}$ and GAA in immature cultures. While $1 \mu \mathrm{M}$ ASA appeared stimulatory on the levels of Arg, $\mathrm{Cr}$ and GAA, suggesting a stimulation of the $\mathrm{Cr}$ synthesis pathway, higher ASA concentration induced a dose-dependent decrease of $\mathrm{Arg}, \mathrm{Cr}$ and GAA. $5 \mathrm{mM} \mathrm{NH}_{4} \mathrm{Cl}$ co-exposure prevented the stimulatory effect of low dose ASA $(1 \mu \mathrm{M})$ on $\mathrm{Cr}$, most probably through inhibition of the AGAT enzyme as shown by strong decrease of GAA level. GSA exposure did not show any effect, at any concentrations tested, on $\mathrm{Arg}, \mathrm{Cr}$ and GAA levels in immature cultures. Mean $\pm \mathrm{SEM} ; \mathrm{n}=3$ per replicates in a representative experiment; significance level as compared to controls: ${ }^{+} \mathrm{p}<0.05 /{ }^{++} \mathrm{p}<0.01 /{ }^{+++} \mathrm{p}<0.001$; significance level of $\mathrm{Cr}$ supplementation compared to the respective conditions of $\mathrm{ASA}+\mathrm{NH}_{4} \mathrm{Cl}$ exposure without creatine: $* * * p<0.001$ (Student's T test). 


\section{References}

Aoyagi K, Akiyama K, Shahrzad S, Tomida C, Hirayama A, Nagase S, Takemura K, Koyama A, Ohba S, Narita M (1999) Formation of guanidinosuccinic acid, a stable nitric oxide mimic, from argininosuccinic acid and nitric oxide-derived free radicals. Free Radic Res 31: 59-65.

Arias A, Garcia-Villoria J, Ribes A (2004) Guanidinoacetate and creatine/creatinine levels in controls and patients with urea cycle defects. Mol Genet Metab 82: 220-223.

Balmer C, Pandey AV, Rüfenacht V, Nuoffer JM, Fang P, Wong LJ, Häberle J (2014) Mutations and Polymorphisms in the Human Argininosuccinate Lyase (ASL) Gene. Hum Mutat 35: 27-35.

Baruteau J, Jameson E, Morris AA, Chakrapani A, Santra S, Vijay S, Kocadag H, Beesley CE, Grunewald S, Murphy E, Cleary M, Mundy H, Abulhoul L, Broomfield A, Lachmann R, Rahman Y, Robinson PH, MacPherson L, Foster K, Chong WK, Ridout DA, Bounford KM, Waddington SN, Mills PB, Gissen P, Davison JE (2017) Expanding the phenotype in argininosuccinic aciduria: need for new therapies. $J$ Inherit Metab Dis 40: 357-368.

Bizzoco E, Faussone-Pellegrini MS, Vannucchi MG (2007) Activated microglia cells express argininosuccinate synthetase and argininosuccinate lyase in the rat brain after transient ischemia. Exp Neurol 208: 100-109.

Braissant $O$ (2010) Ammonia toxicity to the brain: effects on creatine metabolism and transport and protective roles of creatine. Mol Genet Metab 100 Suppl 1: S53-S58.

Braissant O, Béard E, Torrent C, Henry H (2010) Dissociation of AGAT, GAMT and SLC6A8 in CNS: relevance to creatine deficiency syndromes. Neurobiol Dis 37: 423433.

Braissant O, Cagnon L, Monnet-Tschudi F, Speer O, Wallimann T, Honegger P, Henry H (2008) Ammonium alters creatine transport and synthesis in a 3D culture of developing brain cells, resulting in secondary cerebral creatine deficiency. Eur $\mathbf{J}$ Neurosci 27: 1673-1685.

Braissant O, Gotoh T, Loup M, Mori M, Bachmann C (1999) L-arginine uptake, the citrulline-NO cycle and arginase II in the rat brain: An in situ hybridization study. Mol Brain Res 70: 231-241.

Braissant O, Henry H, Villard AM, Zurich MG, Loup M, Eilers B, Parlascino G, Matter E, Boulat O, Honegger P, Bachmann C (2002) Ammonium-induced impairment of axonal growth is prevented through glial creatine. J Neurosci 22: 9810-9820.

Braissant O, Honegger P, Loup M, Iwase K, Takiguchi M, Bachmann C (1999) Hyperammonemia: regulation of argininosuccinate synthetase and argininosuccinate lyase genes in aggregating cell cultures of fetal rat brain. Neurosci Lett 266: 89-92.

Cagnon L, Braissant O (2008) Role of caspases, calpain and cdk5 in ammonia-induced cell death in developing brain cells. Neurobiol Dis 32: 281-292.

Cagnon L, Braissant O (2009) CNTF protects oligodendrocytes from ammonia toxicity: intracellular signaling pathways involved. Neurobiol Dis 33: 133-142. 
Diserens G, Vermathen M, Zurich MG, Vermathen P (2018) Longitudinal investigation of the metabolome of 3D aggregating brain cell cultures at different maturation stages by ${ }^{1}$ H HR-MAS NMR. Anal Bioanal Chem 410: 6733-6749.

Erez A (2013) Argininosuccinic aciduria: from a monogenic to a complex disorder. Genet Med 15: 251-257.

Erez A, Nagamani SC, Shchelochkov OA, Premkumar MH, Campeau PM, Chen Y, Garg HK, Li L, Mian A, Bertin TK, Black JO, Zeng H, Tang Y, Reddy AK, Summar M, O'Brien WE, Harrison DG, Mitch WE, Marini JC, Aschner JL, Bryan NS, Lee B (2011) Requirement of argininosuccinate lyase for systemic nitric oxide production. Nat Med 17: 1619-1626.

Häberle J, Rubio V (2014) Hyperammonemias and related disorders. In: Blau N, Duran M, Gibson KM, Dionisi-Vici C (eds) Physician's guide to the diagnosis, treatment, and follow-up of inherited metabolic diseases. Springer, pp 47-62.

Hanna-El-Daher L, Béard E, Henry H, Tenenbaum L, Braissant O (2015) Mild guanidinoacetate increase under partial guanidinoacetate methyltransferase deficiency strongly affects brain cell development. Neurobiol Dis 79: 14-27.

Jafari P, Braissant O, Zavadakova P, Henry H, Bonafé L, Ballhausen D (2013a) Ammonium accumulation and cell death in a rat $3 \mathrm{D}$ brain cell model of glutaric aciduria type $\mathrm{I}$. PLoS One 8: e53735.

Jafari P, Braissant O, Zavadakova P, Henry H, Bonafé L, Ballhausen D (2013b) Brain damage in methylmalonic aciduria: 2-methylcitrate induces cerebral ammonium accumulation and apoptosis in 3D organotypic brain cell cultures. Orphanet J Rare Dis 8: 4.

Nagamani SC, Erez A, Lee B (2012) Argininosuccinate lyase deficiency. Genet Med 14: 501507.

O'Brien WE, Barr RH (1981) Argininosuccinate lyase: purification and characterization from human liver. Biochemistry 20: 2056-2060.

O'Brien WE, McInnes R, Kalumuck K, Adcock M (1986) Cloning and sequence analysis of cDNA for human argininosuccinate lyase. Proc Natl Acad Sci U S A 83: 7211-7215.

Ratner S, Petrack B (1953) The mechanism of arginine synthesis from citrulline in kidney. J Biol Chem 200: 175-185.

Roze E, Azuar C, Menuel C, Haberle J, Guillevin R (2007) Usefulness of magnetic resonance spectroscopy in urea cycle disorders. Pediatr Neurol 37: 222-225.

Rüegger CM, Lindner M, Ballhausen D, Baumgartner MR, Beblo S, Das A, Gautschi M, Glahn EM, Grünert SC, Hennermann J, Hochuli M, Huemer M, Karall D, Kölker S, Lachmann RH, Lotz-Havla A, Möslinger D, Nuoffer JM, Plecko B, Rutsch F, Santer R, Spiekerkoetter U, Staufner C, Stricker T, Wijburg FA, Williams M, Burgard P, Häberle J (2014) Cross-sectional observational study of 208 patients with nonclassical urea cycle disorders. J Inherit Metab Dis 37: 21-30.

Sampaleanu LM, Vallee F, Thompson GD, Howell PL (2001) Three-dimensional structure of the argininosuccinate lyase frequently complementing allele Q286R. Biochemistry 40: $15570-15580$. 
Simard L, O'Brien WE, McInnes RR (1986) Argininosuccinate lyase deficiency: evidence for heterogeneous structural gene mutations by immunoblotting. Am J Hum Genet 39: $38-51$.

Summar ML, Koelker S, Freedenberg D, Le Mons C, Häberle J, Lee HS, Kirmse B (2013) The incidence of urea cycle disorders. Mol Genet Metab 110: 179-180.

Tomlinson S, Westall RG (1964) Argininosuccinic Aciduria. Argininosuccinase and Arginase in Human Blood Cells. Clin Sci 26: 261-269.

Turner MA, Simpson A, McInnes RR, Howell PL (1997) Human argininosuccinate lyase: a structural basis for intragenic complementation. Proc Natl Acad Sci U S A 94: 90639068.

van Spronsen FJ, Reijngoud DJ, Verhoeven NM, Soorani-Lunsing RJ, Jakobs C, Sijens PE (2006) High cerebral guanidinoacetate and variable creatine concentrations in argininosuccinate synthetase and lyase deficiency: implications for treatment? Mol Genet Metab 89: 274-276.

Wu G, Borbolla AG, Knabe DA (1994) The uptake of glutamine and release of arginine, citrulline and proline by the small intestine of developing pigs. J Nutr 124: 24372444. 

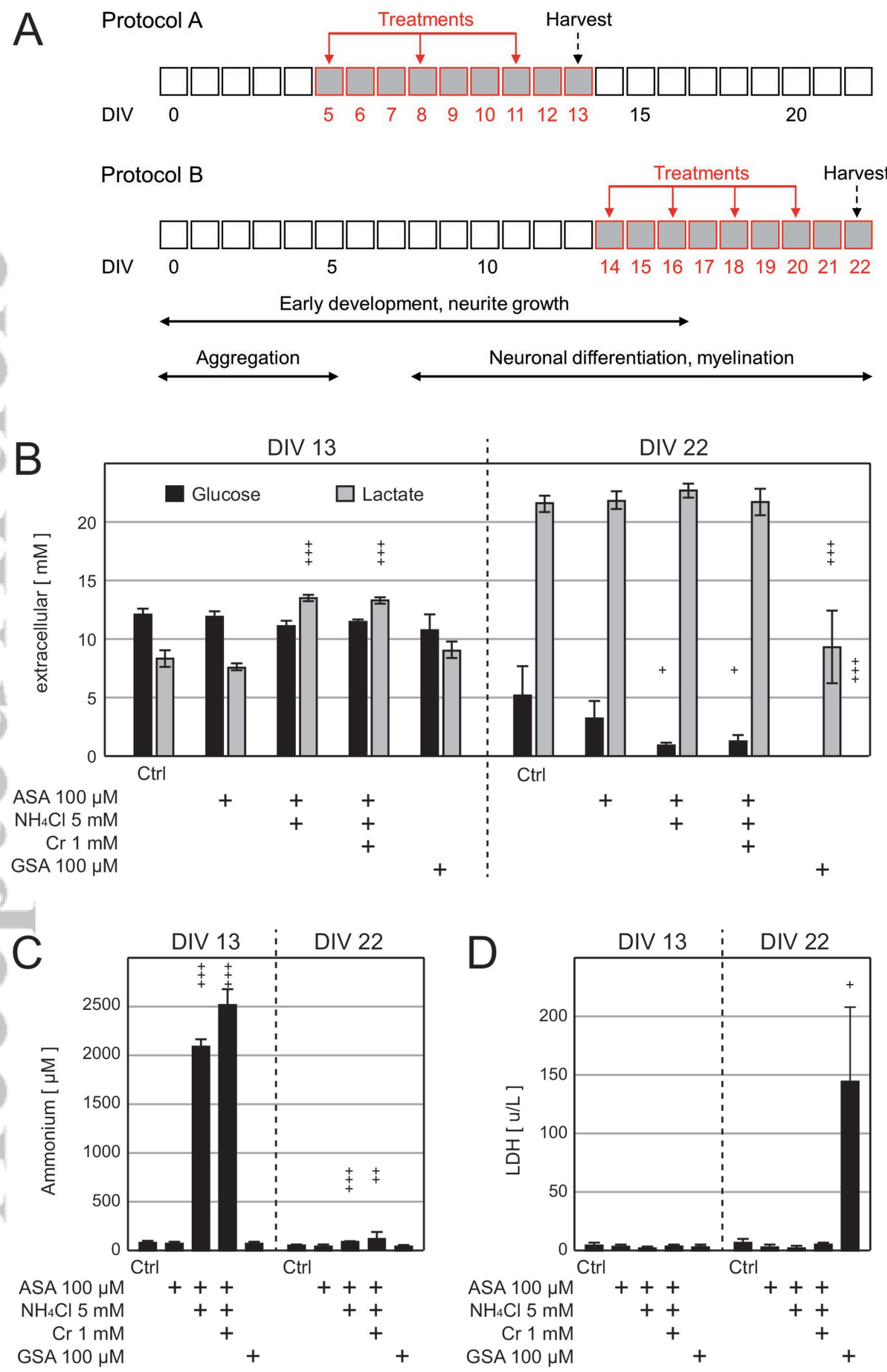

Diez-Fernandez et al, Figure 1 jimd_12090_boli-d-18-00380 diez jimd figure 1 revised 190304.eps 
A Developing neurons (DIV 13 / NFM \& p-NFM)

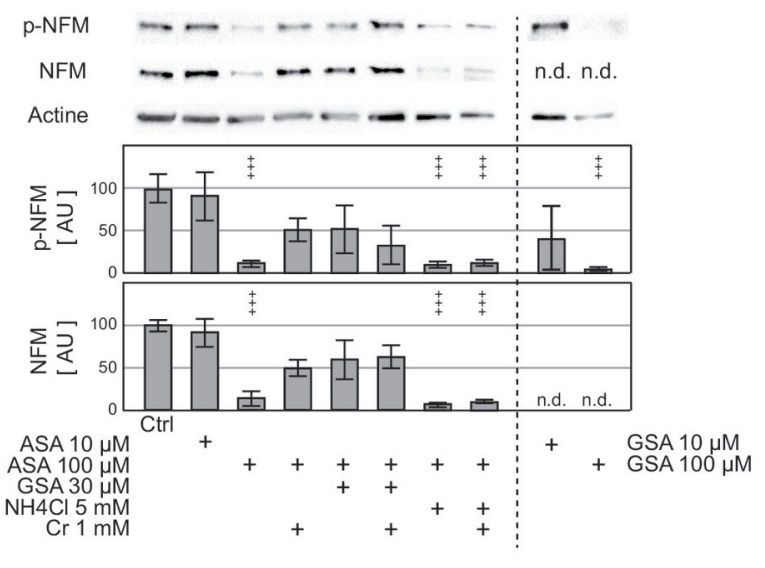

Mature neurons (DIV 22 / NFM \& p-NFM)

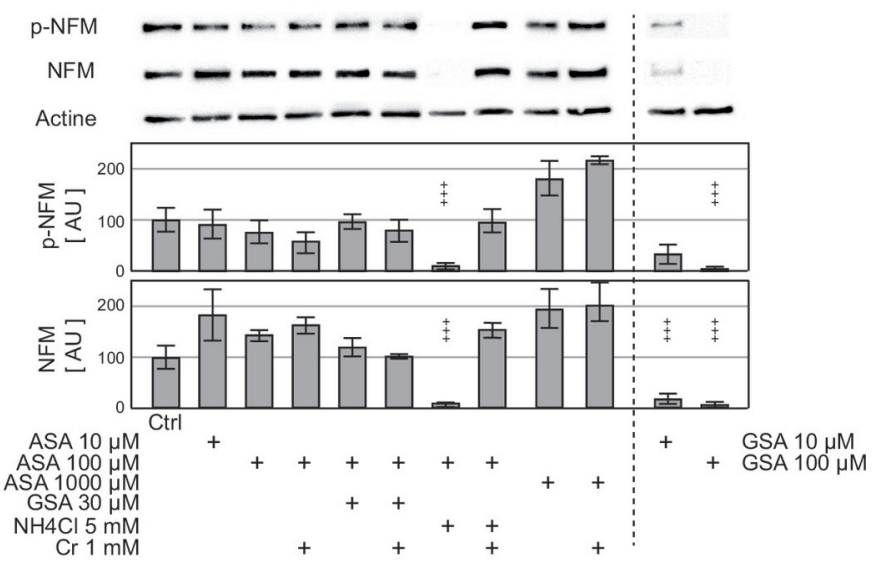

B Astrocytes (GFAP)

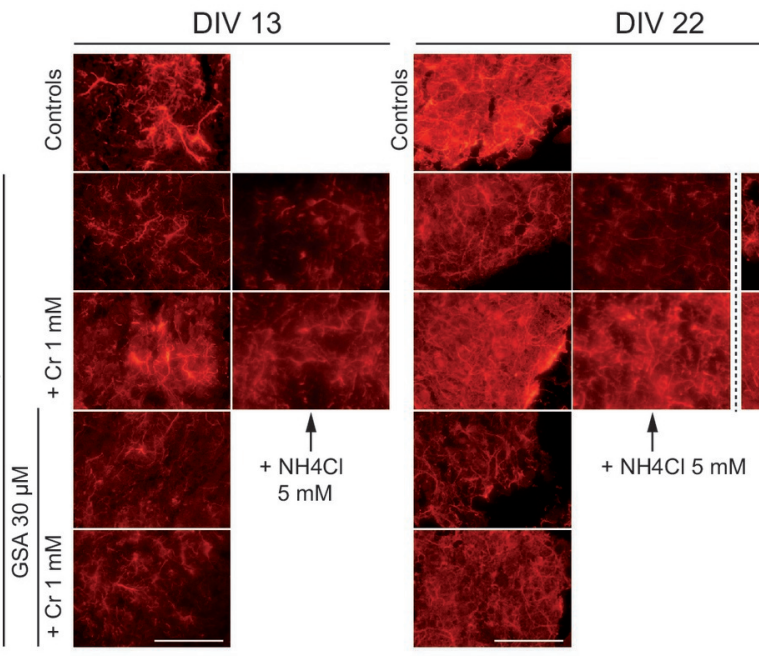

ASA $1 \mathrm{mM}$

$\downarrow$

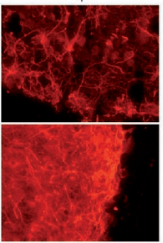

GFAP $-m-\infty=$

DIV 22

Actin -

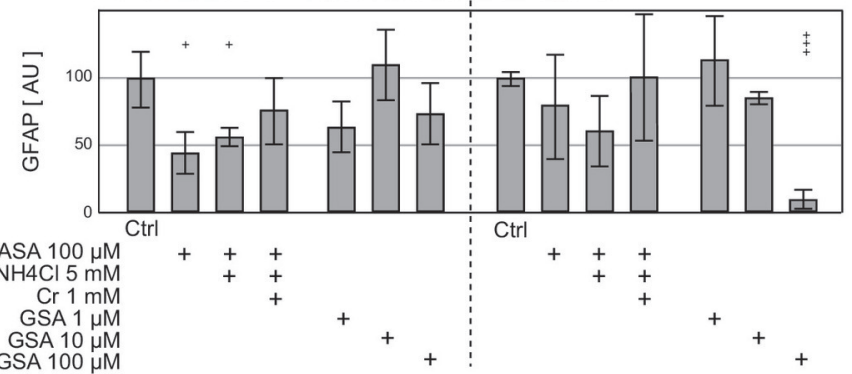

C Oligodendrocytes (MBP)

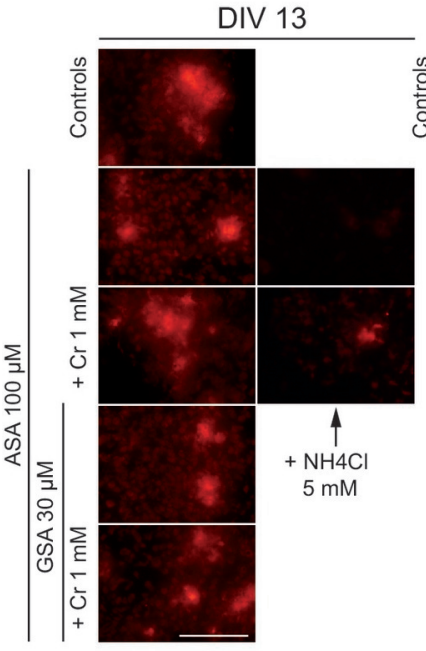

DIV 22

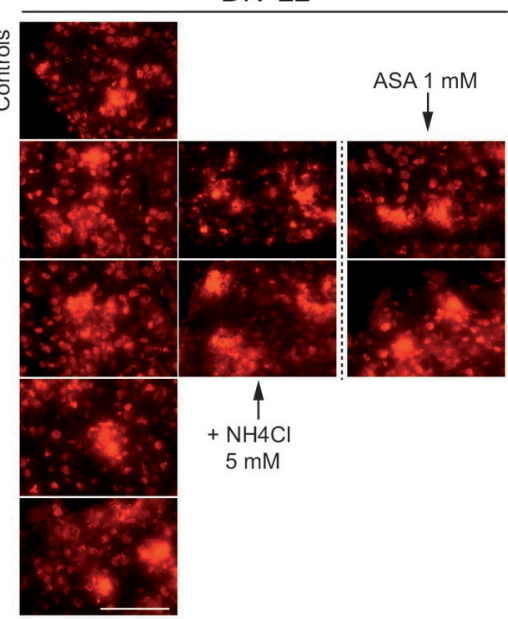

Diez-Fernandez et al, Figure 2 jimd_12090_boli-d-18-00380 diez jimd figure 2 revised.eps 
A Apoptosis (Cleaved caspase 3)
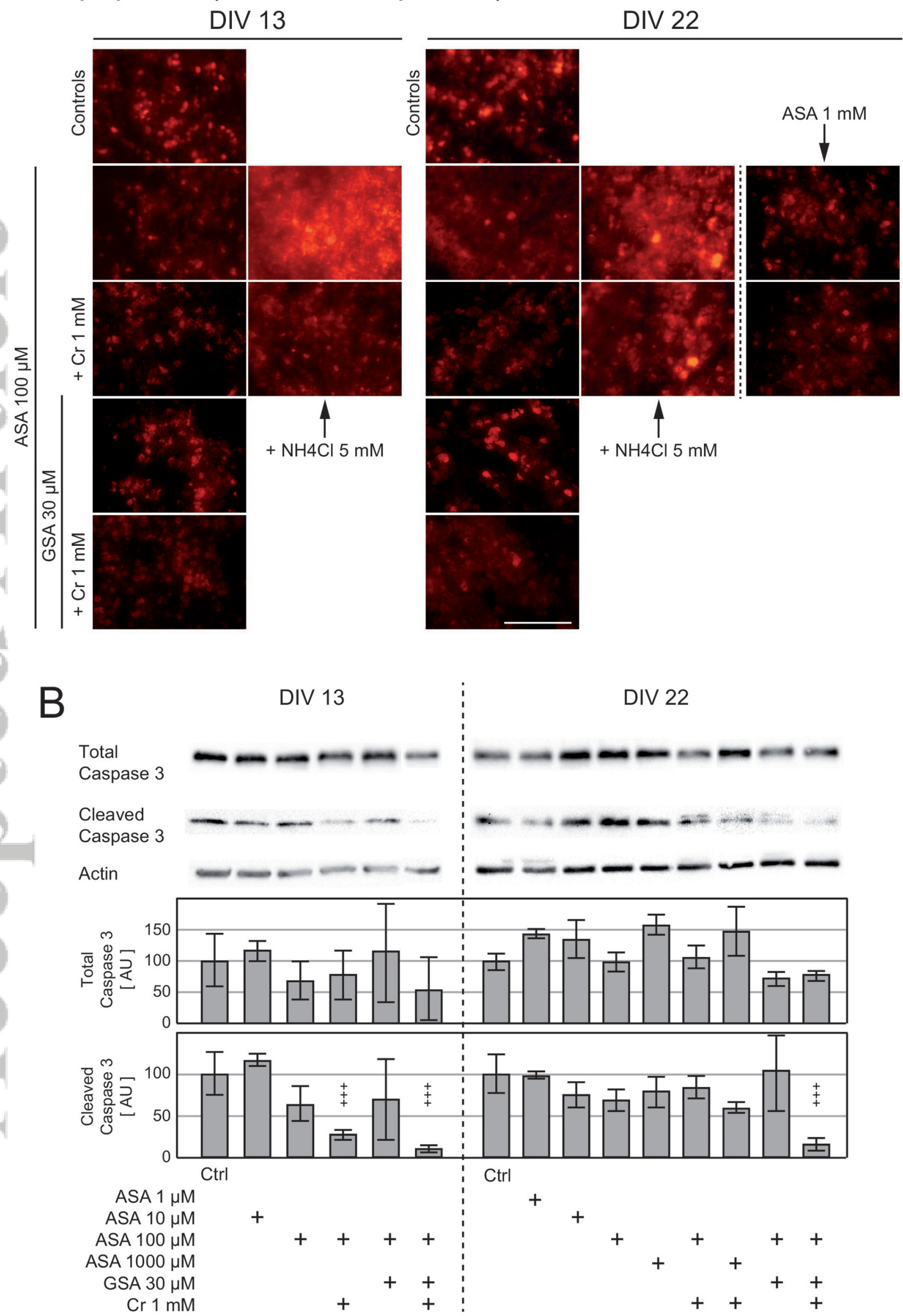

Diez-Fernandez et al, Figure 3 Apoptosis jimd_12090_boli-d-18-00380 diez jimd figure 3 revised.eps 

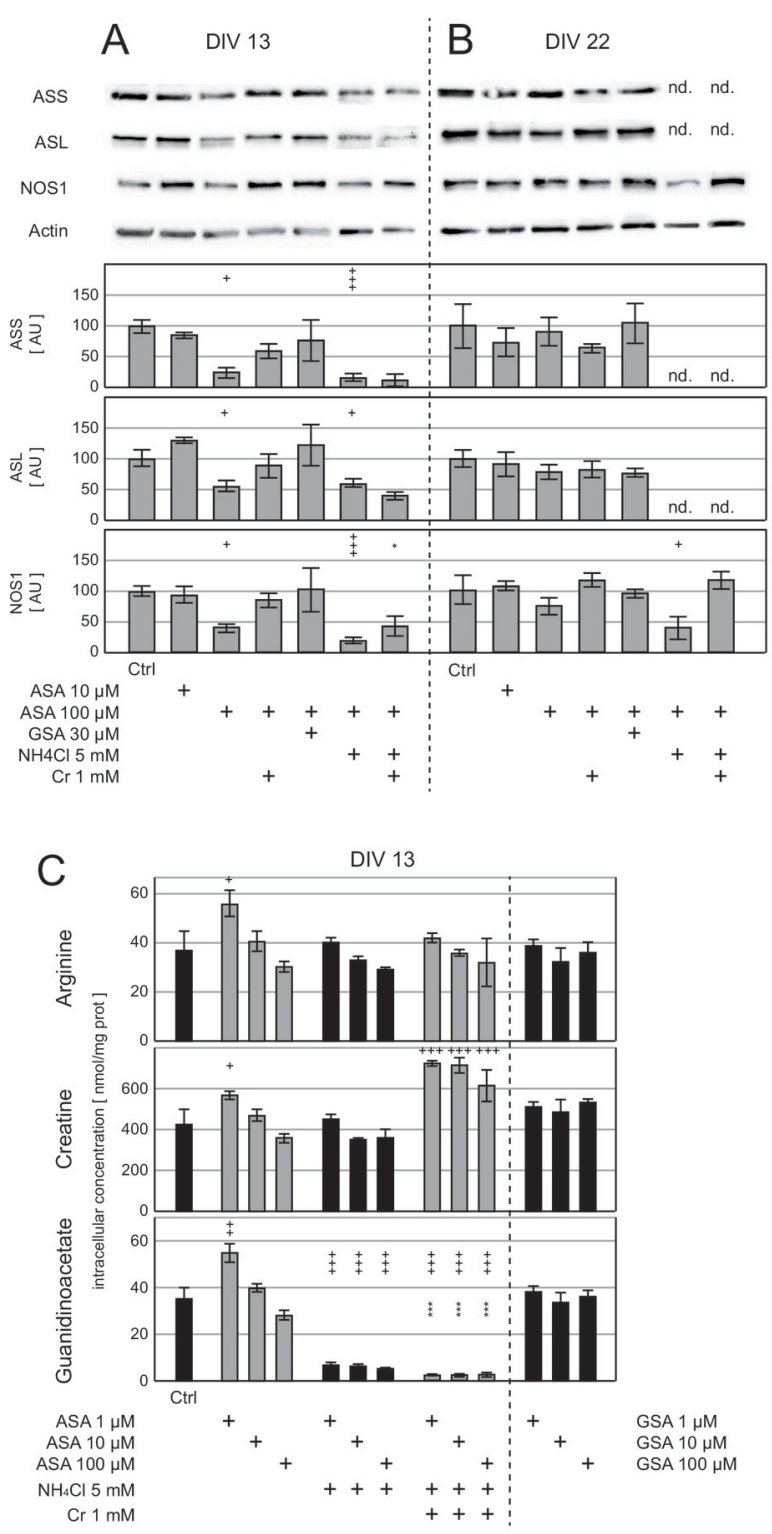

Diez-Fernandez et al, Figure 4

jimd_12090_boli-d-18-00380 diez jimd figure 4 revised.eps 